\title{
On Some Expansions for Bessel Integral Functions
}

\section{F. Oberhettinger ${ }^{1}$}

\begin{abstract}
Investigations by van der Pol and Humbert concerning the Bessel integral function of order zero are extended to Bessel functions of other kinds and to functions related to Bessel functions.
\end{abstract}

The Bessel integral function of order zero,

$$
J i_{0}(x)=-\int_{x}^{\infty} t^{-1} J_{0}(t) d t
$$

was introduced by van der Pol [8]. ${ }^{2}$ Equivalent $[3,4,6]$ with (1) is

$$
J i_{0}(x)=\frac{1}{\pi} \int_{0}^{\pi} C i(x \sin t) d t
$$

Various properties of this function have been investigated by Humbert [4]. A number of definite integrals, especially of the Fourier or Laplace transform type, can be reduced to (1). For this reason tables of $J i_{0}(x)$ have been published previously [5]. The numerical computation of $J_{0}(x)$ requires the transformation of the integral in (1) into an expression involving a series in either ascending powers (for not too large $x$ ) or descending powers (for large $x$ ) of the variable $x$. One of these expansions $[4,6]$ is

where one can write

$$
J i_{0}(x)=\gamma+\log \frac{x}{2}+\sum_{n=1}^{\infty} \frac{(-1)^{n}(x / 2)^{2 n}}{2 n(n !)^{2}},
$$

$$
\sum_{n=1}^{\infty} \frac{(-1)^{n}(x / 2)^{2 n}}{2 n(n !)^{2}}=\int_{0}^{x} t^{-1}\left[J_{0}(t)-1\right] d t .
$$

An asymptotic expansion of $J i_{0}(x)$ for large $x[5,7]$ is listed in (6). The preparation of a set of numerical tables by the Computation Laboratory made it necessary to derive expansions of types (3) and (6) for functions defined similarly to (1) but with $J_{0}(t)$ replaced by another kind of Bessel or related function. The established results are listed below. (A list of notations and auxiliary formulas is given at the end of this paper.)

$$
\begin{aligned}
& \frac{\pi}{2} Y i_{0}(x)=-\frac{\pi}{2} \int_{x}^{\infty} t^{-1} Y_{0}(t) d t=\frac{1}{2}\left(\log \frac{x}{2}\right)^{2}+\gamma \log \frac{x}{2}+\frac{\gamma^{2}}{2}-\frac{\pi^{2}}{12}-\sum_{n=1}^{\infty} \frac{(-1)^{n}(x / 2)^{2 n}}{2 n(n !)^{2}}\left[\psi(n+1)+\frac{1}{2 n}-\log \frac{x}{2}\right] \\
& K i_{0}(x)=-\int_{x}^{\infty} t^{-1} K_{0}(t) d t=-\frac{1}{2}\left(\log \frac{x}{2}\right)^{2}-\gamma \log \frac{x}{2}-\frac{\gamma^{2}}{2}-\frac{\pi^{2}}{24}+\sum_{n=1}^{\infty} \frac{(x / 2)^{2 n}}{2 n(n !)^{2}}\left[\psi(n+1)+\frac{1}{2 n}-\log \frac{x}{2}\right] .
\end{aligned}
$$

Both these expressions correspond to (3) and are suitable for not too large $x$.

For large $x$ we have the asymptotic expansions of the following functions:

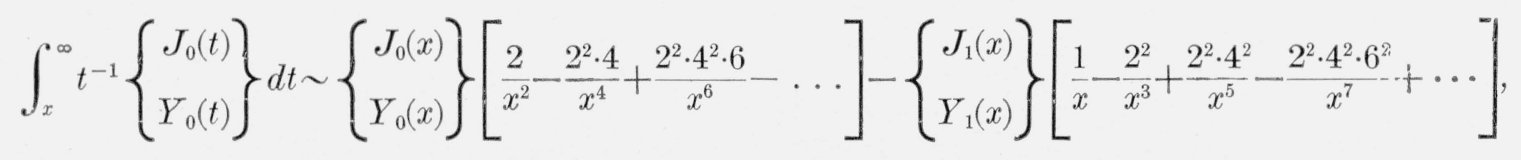

1 Guest worker, National Bureau of Standards, from The American University,

${ }^{2}$ Figures in brackets indicate the literature references at the end of this paper. 


$$
\begin{aligned}
& \int_{x}^{\infty} t^{-1} K_{0}(t) d t \sim-K_{0}(x)\left[\frac{2}{x^{2}}+\frac{2^{2} \cdot 4}{x^{4}}+\frac{2^{2} \cdot 4^{2} \cdot 6}{x^{6}}+\cdots\right]+K_{1}(x)\left[\frac{1}{x}+\frac{2^{2}}{x^{3}}+\frac{2^{2} \cdot 4^{2}}{x^{5}}+\frac{2^{2} \cdot 4^{2} \cdot 6^{2}}{x^{7}}+\cdots\right], \\
& \int_{x}^{\infty} t^{-1}\left[\boldsymbol{H}_{0}(t)-Y_{0}(t)\right] d t \sim \frac{2}{\pi x} \sum_{n=0}^{\infty}(-1)^{n}\left[\frac{(2 n) !}{n !}\right]^{2} \frac{(2 x)^{-2 n}}{2 n+1}, \\
& \int_{0}^{x}\left[\boldsymbol{H}_{0}(t)-Y_{0}(t)\right] d t-\frac{2}{\pi} \log (2 x)-\frac{2}{\pi} \gamma \sim-\frac{2}{\pi} \sum_{n=1}^{\infty}(-1)^{n} \frac{(2 n) !(2 n-1) !}{(n !)^{2}}(2 x)^{-2 n}, \\
& \int_{0}^{x}\left[I_{0}(t)-\boldsymbol{L}_{0}(t)\right] d t-\frac{2}{\pi} \log (2 x)-\frac{2}{\pi} \gamma \sim-\frac{2}{\pi} \sum_{n=1}^{\infty} \frac{(2 n) !(2 n-1) !}{(n !)^{2}}(2 x)^{-2 n} .
\end{aligned}
$$

Fuithermore, we derive the identity

$$
\int_{x}^{\infty} t^{-2} \boldsymbol{H}_{1}(t) d t=(2 x)^{-1} \boldsymbol{H}_{1}(x)+\frac{1}{2} \int_{x}^{\infty} t^{-1} \boldsymbol{H}_{0}(t) d t .
$$

Note that with (6) we know the asymptotic expansion of $\int_{x}^{\infty} t^{-1} \boldsymbol{H}_{0}(t) d t$ for large $x$ by virtue of (8). With this and the known asymptotic expansion of $\boldsymbol{H}_{1}(x)$ (see (21)), the asymptotic expansion of (11) is likewise known.

a. Proof of (4) and (5). We write

$$
\int_{x}^{\infty} t^{-1} J_{\nu}(t) d t=\int_{0}^{\infty} t^{-1} J_{\nu}(t) d t-\int_{0}^{x} t^{-1} J_{\nu}(t) d t
$$

and since (using term-by-term integration)

$$
\int_{0}^{x} t^{-1} J_{v}(t) d t=\sum_{m=0}^{\infty} \frac{\left(-1^{m}(x / 2)^{\nu+2 m}\right.}{m ! \Gamma(\nu+m+1)(\nu+2 m)},
$$

we obtain by (23)

$$
\int_{x}^{\infty} t^{-1} J_{\nu}(t) d t=\frac{1}{\nu}\left[1-\frac{(x / 2)^{\nu}}{\Gamma(1+\nu)}\right]-\sum_{m=1}^{\infty} \frac{(-1)^{m}(x / 2)^{\nu+2 m}}{m ! \Gamma(\nu+m+1)(\nu+2 m)} .
$$

This defines an integral function of $\nu$. If we now let $\nu$ tend to zero, using (13) we obtain the expansion (3). In ordei to prove (4), we differentiate the preceeding formula with respect to $\nu$. If we again let $\nu$ tend to zero, using (12) and (13) we obtain (4). Finally, the relation

$$
K_{0}(t)=\frac{1}{2} i \pi\left[J_{0}(i t)+i Y_{0}(i t)\right]
$$

together with (3) and (4), yields (5).

b. Proof of (6) and (7). We replace the Lommel functions in (24) by their asymptotic expansion (22), and the results in (6) follow immediately. Again, the relation

$$
K_{0}(t)=\frac{1}{2} i \pi\left[J_{0}(i t)+i Y_{0}(i t)\right],
$$

together with (6), yields (7). (Note that the asymptotic expansion of

$$
\int_{x}^{\infty} t^{u}\left\{\begin{array}{l}
J_{\nu}(t) \\
Y_{\nu}(t) \\
K_{\nu}(t)
\end{array}\right\} d t
$$

can be obtained in the same way.) 
c. Proof of (8). From (21) we have, for $\nu=0$ and large $t$,

$$
t^{-1}\left[\boldsymbol{H}_{0}(t)-Y_{0}(t)\right] \sim \frac{2}{\pi} \sum_{n=0}^{\infty}(-1)^{n} 2^{-2 n}\left[\frac{(2 n) !}{n !}\right]^{2} t^{-2 n-2} .
$$

Integrating term by term, we obtain (8).

d. Proof of (9). We use (19) and obtain, interchanging the order of integration,

$$
\int_{0}^{x}\left[\boldsymbol{H}_{0}(t)-Y_{0}(t)\right] d t=\frac{2}{\pi} \int_{0}^{\infty} v^{-1}\left(1+v^{2}\right)^{-1 / 2}\left(1-e^{-x v}\right) d v .
$$

Because it is necessary to represent the 1 . h. s. function of $x$ as an integral of the Laplace transform type in order to apply Watson's lemma [1] for the determination of its asymptotic expansion, we form, using (14),

$$
\int_{0}^{x}\left[\boldsymbol{H}_{0}(t)-Y_{0}(t)\right] d t-\frac{2}{\pi} \log x=\frac{2}{\pi} \int_{0}^{\infty} v^{-1}\left[\left(1+v^{2}\right)^{-1 / 2}-e^{-v}\right) d v+\frac{2}{\pi} \int_{0}^{\infty} e^{-x v} v^{-1}\left[1-\left(1+v^{2}\right)^{-1 / 2}\right] d v .
$$

The first integral on the r. h. s. is a constant equal to $\gamma+\log 2$. The second integral is of the Laplace transform type and can easily be developed asymptotically for large $x$. We obtain first

$$
\begin{aligned}
\int_{0}^{\infty} v^{-1}\left[\left(1+v^{2}\right)^{-1 / 2}-e^{-v}\right] d_{\prime \prime} & =\lim _{\delta \rightarrow 0}\left[\int_{\delta}^{\infty} v^{-1}\left(1+v^{2}\right)^{-1 / 2} d v-\int_{\delta}^{\infty} v^{-1} e^{-v} d v\right] \\
& =\lim _{\delta \rightarrow 0}\left\{\operatorname{Ei}(-\delta)-\log \left[\frac{1+\left(1+v^{2}\right)^{1 / 2}}{v}\right]_{\delta}\right\} \\
& =\gamma+\log 2, \quad \text { by }(15) .
\end{aligned}
$$

The asymptotic expansion of the second integral for large $x$ is as follows.

We expand

$$
v^{-1}\left[1-\left(1+v^{2}\right)^{-1 / 2}\right]=-\sum_{n=1}^{\infty} \frac{(-1)^{n}(2 n) ! 2^{-2 n} v^{2 n-1}}{(n !)^{2}} .
$$

The application of Watson's lemma [1] gives (9).

e. Proof of (10). We use (20) to obtain

$$
\int_{0}^{x}\left[I_{0}(t) \boldsymbol{L}_{0}(t)\right] d t=\frac{2}{\pi} \int_{0}^{1} v^{-1}\left(1-v^{2}\right)^{-1 / 2}\left(1-e^{-v x}\right) d v,
$$

and proceed precisely as before, arriving at the intermediate result

$$
\int_{0}^{x}\left[I_{0}(t)-L_{0}(t)\right] d i-\frac{2}{\pi} \log (2 x)-\frac{2}{\pi} \gamma=\frac{2}{\pi}\left\{-E i(-x)+\int_{0}^{1} v^{-1} e^{-v x}\left[1-\left(1-v^{2}\right)^{-1 / 2}\right] d v\right\} .
$$

We expand

$$
v^{-1}\left[1-\left(1-v^{2}\right)^{-1 / 2}\right]=-\sum_{n=1}^{\infty} \frac{2^{-2 n}(2 n) ! v^{2 n-1}}{(n !)^{2}} .
$$

The application of Watson's lemma [1] gives (10). (Note that the term $-E i(-x)$ is purely formal and may be omitted, because its asymptotic expansion consists of the product of an exponential function with negative exponent and a series in descending powers of $x$.)

f. Proof of (11). By means of (18) we obtain

$$
\int_{x}^{\infty} t^{-2} \boldsymbol{H}_{1}(t) d t=-\frac{2}{\pi} \int_{0}^{1}\left(1-v^{2}\right)^{1 / 2} \operatorname{si}(v x) d v
$$


By partial integration,

$$
\int_{x}^{\infty} t^{-2} \boldsymbol{H}_{1}(t) d t=-\frac{1}{2} \operatorname{si}(x)+\frac{1}{2} x^{-1} \boldsymbol{H}_{1}(x)+\frac{1}{\pi} \int_{0}^{1} v^{-1} \sin ^{-1} v \sin (v x) d v
$$

but

$$
\int_{0}^{1} v^{-1} \sin ^{-1} v \sin (v x) d v=\frac{1}{2} \pi \operatorname{si}(x)+\frac{1}{2} \pi \int_{x}^{\infty} t^{-1} \boldsymbol{H}_{0}(t) d t
$$

and (11) follows at once.

Notations:

$J_{\nu}(t)=$ Bessel function of the first kind.

$Y_{\nu}(t)=$ Bessel function of the second kind.

$I_{\nu}(t)=$ modified Bessel function of the first kind.

$K_{\nu}(t)=$ modified Bessel function of the second kind.

$\boldsymbol{H}_{\nu}(t)=$ Struve's function.

$\boldsymbol{L}_{\nu}(t)=-i e^{-i v \cdot \pi / 2} \boldsymbol{H}_{\nu}(i t)$.

All these functions are Bessel or related functions of the order $\nu$.

$\Gamma^{\prime}(z)=$ Gamma function.

$\psi(z)=\Gamma^{\prime}(z) / \Gamma(z)$.

$\gamma=-\psi(1)=0.577215 \ldots$. . . (Euler's constant).

$S \mu, \nu(z)=$ Lommel's function.

$\left[\frac{\partial J_{\nu}(z)}{\partial \nu}\right]_{\nu=0}=\frac{\pi}{2} Y_{0}(z) \quad[9$, p. 61,62$]$

$$
\frac{1}{\Gamma(1+z)}=1+\gamma z+\left(\gamma^{2}-\frac{\pi^{2}}{6}\right) \frac{z^{2}}{2}+O\left(z^{3}\right)
$$

(Taylor expansion of $1 / \Gamma(1+z)$ near $z=0)$.

Integral representations:

$$
\begin{aligned}
& \log x=\int_{0}^{\infty} v^{-1}\left(e^{-v}-e^{-x v}\right) d v \quad[2, \text { p. 17] } \\
& \operatorname{Ei}(-\delta)=-\int_{\delta}^{\infty} v^{-1} e^{-v} d v \\
& \operatorname{Ci}(\delta)=-\int_{\delta}^{\infty} v^{-1} \cos v d v \\
& \operatorname{si}(\delta)=-\int_{\delta}^{\infty} v^{-1} \sin v d v .
\end{aligned}
$$

The last three functions are the exponential integral, the cosine integral, and the sine integral, respectively.

$$
\begin{aligned}
& \Gamma\left(\nu+\frac{1}{2}\right) \boldsymbol{H}_{\nu}(t)=2 \pi^{-1 / 2}\left(\frac{1}{2} t\right)^{\nu} \int_{0}^{1}\left(1-v^{2}\right)^{\nu-(1 / 2)} \sin (v t) d v, \quad \operatorname{Re} \nu>-\frac{1}{2} \quad[9, \mathrm{p} .328] \\
& \boldsymbol{H}_{0}(t)-Y_{0}(t)=\frac{2}{\pi} \int_{0}^{\infty} e^{-t v}\left(1+v^{2}\right)^{-1 / 2} d v \quad[9, \mathrm{p} .331] \\
& I_{0}(t)-\boldsymbol{L}_{0}(t)=\frac{2}{\pi} \int_{0}^{1} e^{-t v}\left(1-v^{2}\right)^{-1 / 2} d v .
\end{aligned}
$$




$$
\begin{aligned}
& \boldsymbol{H}_{\nu}(z)-Y_{\nu}(z) \sim \pi^{-3 / 2} \cos (\pi \nu)\left(\frac{1}{2} z\right)^{\nu-1} \sum_{n=0}^{\infty} \frac{(-1)^{n} \Gamma\left(\frac{1}{2}-\nu+n\right)(2 n) !}{n ! z^{2 n}} \quad[9, \text { p. 333] } \\
& S_{\mu, \nu}(z) \sim z^{\mu-1}\left\{1-\frac{(\mu-1)^{2}-\nu^{2}}{z^{2}}+\frac{\left.\left[(\mu-1)^{2}-\nu^{2}\right][\mu-3)^{2}-\nu^{2}\right]}{z^{4}}-\cdots\right\} \cdot \quad[9, \text { p. 351] }
\end{aligned}
$$

\section{Integral formulas:}

$$
\begin{aligned}
& \int_{0}^{\infty} t^{\mu-1} J_{\nu}(t) d t=2^{\mu-1} \Gamma\left(\frac{1}{2}+\frac{1}{2} \mu\right) / \Gamma\left(1+\frac{1}{2} \nu-\frac{1}{2} \mu\right), \quad-\operatorname{Re} \nu<\operatorname{Re} \mu<\frac{3}{2} \quad[9, \text { p. 391] } \\
& \int_{x}^{\infty} t^{-1}\left\{\begin{array}{l}
J_{0}(t) \\
Y_{0}(t)
\end{array}\right\} d t=2 x\left\{\begin{array}{l}
J_{0}(x) \\
Y_{0}(x)
\end{array}\right\} S_{-2,1}(x)-x\left\{\begin{array}{l}
J_{1}(x) \\
Y_{1}(x)
\end{array}\right\} S_{-1,0}(x) . \quad[9, \text { p. 350] }
\end{aligned}
$$

[1] E. T. Copson, Theory of a complex variable, p. 218 (Oxford Univ. Press, London, 1946).

[2] A. Erdelyi, Higher transcendental functions, vol. 1, Bateman Manuscript Project, Calif. Inst. Technol. (McGraw-Hill Book Co., Inc., New York, N. Y., 1953).

[3] P. Humbert, Sur les fonctions de Bessel integrales, Compt. rend. 195, 854-855 (1932).

[4] P. Humbert, Bessel integral functions, Proc. Edinburgh Math. Soc. 3, 276-285 (1933).

[5] A. N. Lowan, G. Blanch, and M. Abramowitz, Table of $J i_{0}(\mathrm{x})=\int_{x}^{\infty} J_{0}(t) / t d t$ and related functions, J. Math. Physies 22, 51-57 (1943).

[6] G. Petiau, La theorie des fonctions de Bessel, p. 251-258 (Centre National de la Recherche Scientifique, Paris, 1955).

[7] V. G. Smith, An asymptotic expansion of $J i_{0}(x)=-\int_{x}^{\infty} J_{0}(t) / t d t$, J. Math. Physics 22, 58-59 (1943).

[8] B. van der Pol, On the operational solution of linear differential equations and an investigation of the properties of their solutions, Phil. Mag. 8, 861-898 (1929).

[9] G. N. Watson, Bessel functions (Cambridge Univ. Press, England, 1944).

Washington, February 27, 1957. 